\title{
CSF IgA NMDAR antibodies are potential biomarkers for teratomas in anti-NMDAR encephalitis \\ OPEN
}

Virginie Desestret, MD, $\mathrm{PhD}$

Aude Chefdeville, MSc Aurélien Viaccoz, MD Chloe Bost, MSc

François Ducray, MD, $\mathrm{PhD}$

Géraldine Picard

Veronique Rogemond, $\mathrm{PhD}$

Marie-Oceane Chaffois Charlotte Blanc, MSc

Claire Bardel, PhD

Isabelle Treilleux, MD,

$\mathrm{PhD}$

Olivier Pascual, PhD

Jean-Christophe Antoine, MD

Jean-Yves Delattre, MD

Jerome Honnorat, MD, $\mathrm{PhD}$

Correspondence to

Dr. Honnorat:

jerome.honnorat@chu-lyon.fr

Supplemental data at Neurology.org/nn

\section{ABSTRACT}

Objective: To evaluate the presence of immunoglobulin A (IgA) subtype of anti-NMDA receptor (NMDAR) antibodies (IgA-NMDAR-Abs) in the CSF of patients with immunoglobulin G (IgG)NMDAR-Ab encephalitis and to describe the potential association with a specific clinical pattern.

Methods: The retrospective analysis for the presence of IgA-NMDAR-Abs in 94 CSF samples from patients with anti-NMDAR encephalitis diagnosed between October 2007 and February 2014 was conducted at the French Reference Centre on Paraneoplastic Neurological Syndrome. This observational study compared 39 patients with both IgA- and IgG-NMDAR-Abs to 55 patients with only IgG-NMDAR-Abs.

Results: In the retrospective cohort, $41 \%$ of the patients with NMDAR-Ab encephalitis had both CSF IgG- and IgA-NMDAR-Abs. Approximately half of the IgA-NMDAR-Ab-positive patients (18/ $38,49 \%)$ definitively possessed associated tumors, primarily ovarian teratomas (17/18, 94\%), compared with only $5 \%$ (3/55) of the patients in the IgA-NMDAR-Ab-negative group $(p<0.001)$. In the adult female population at risk for ovarian teratoma, the detection of CSF IgA-NMDAR-Ab positivity showed $85 \%$ sensitivity, $70 \%$ specificity, a $57 \%$ positive predictive value, and a $90 \%$ negative predictive value for the diagnosis of ovarian teratoma. No other specific clinical features or clinical outcome were associated with CSF IgA-NMDAR-Ab positivity.

Conclusion: These results suggest that in patients with IgG-NMDAR-Ab encephalitis, CSF IgANMDAR-Abs could be used as a biological marker for the presence of an ovarian teratoma. Neurol Neuroimmunol Neuroinflamm 2015;2:e166; doi: 10.1212/NXI.0000000000000166

\section{GLOSSARY}

$\mathbf{A b}=$ antibody; $\mathbf{C B A}=$ cell-based assay; $\mathbf{I C U}=$ intensive care unit; IgA = immunoglobulin $\mathrm{A} ; \mathbf{I g G}=$ immunoglobulin $\mathbf{G} ; \mathbf{I g M}=$ immunoglobulin $\mathrm{M}$; IQR = interquartile range; $\mathbf{m R S}=$ modified Rankin Scale; NMDAR = NMDA receptor; $\mathbf{P B S}=$ phosphatebuffered saline; QAlb = albumin quotient; QIg = immunoglobulins quotient.

Anti-NMDA receptor (NMDAR) encephalitis is a severe autoimmune neuropsychiatric disorder characterized by the circulating immunoglobulin $\mathrm{G}(\mathrm{IgG})$ subtype of anti-NMDAR autoantibodies (IgG-NMDAR-Abs). IgG-NMDAR-Abs are directly involved in the pathophysiology of this encephalitis, leading to the selective and reversible internalization of cell-surface NMDARs. ${ }^{1-3}$ Diagnosis is based on the presence of highly specific IgG-NMDAR-Abs in the CSF. ${ }^{4,5}$ Immunoglobulin A (IgA) (IgA-NMDAR-Abs) or immunoglobulin M (IgM) (IgM-NMDAR-Abs) directed against NMDAR have also been reported in the sera of some patients with dementia, schizophrenia, affective disorders, Parkinson disease, stroke, or amyotrophic lateral sclerosis, or in healthy controls, ${ }^{6-8}$ but these findings have an unclear diagnostic value. Some authors suggested

\footnotetext{
From the Lyon Neuroscience Research Center (V.D., A.C., C. Bost, F.D., V.R., C. Blanc, O.P., J.-C.A., J.H.), INSERM U1028/CNRS UMR 5292; Université de Lyon-Université Claude Bernard Lyon 1 (V.D., A.C., C. Bost, F.D., V.R., C. Bardel, O.P., J.H.); the Department of Neurology D (V.D.) and French Reference Center on Paraneoplastic Neurological Syndrome (A.V., C. Bost, F.D., G.P., V.R., M.-O.C., C. Blanc, J.-C.A., J.-Y.D., J.H.), Hospices Civils de Lyon, Hôpital Neurologique, Bron; Service de Biostatistique (C. Bardel), Hospices Civils de Lyon, CNRS UMR 5558; Laboratoire de Biométrie et Biologie Evolutive (C. Bardel), Equipe Biostatistique-Santé, Villeurbanne; the Department of Biopathology (I.T.), Centre de Recherche en Cancérologie de Lyon; Service de Neurologie (J.-C.A.), CHU de Saint-Etienne et Université de Lyon, SaintEtienne; and Service de Neurologie Mazarin (J.-Y.D.), Groupe Hospitalier Pitié-Salpêtrière, APHP, Université Pierre et Marie Curie-Paris 6, Centre de Recherche de l'Institut du Cerveau et de la Moelle Épinière, UMR S975, CNRS, UMR 7225, Paris, France.

Funding information and disclosures are provided at the end of the article. Go to Neurology.org/nn for full disclosure forms. The Article Processing Charge was paid by INSERM-DRLYS-FACTURATION.

This is an open access article distributed under the terms of the Creative Commons Attribution-NonCommercial-NoDerivatives License 4.0 (CC BY-NC-ND), which permits downloading and sharing the work provided it is properly cited. The work cannot be changed in any way or used commercially.
} 
that under some circumstances, IgA-NMDARAbs could be pathogenic and that IgA-NMDARAbs would be present in the serum of $31 \%$ of patients with IgG-NMDAR-Ab encephalitis. ${ }^{6}$

The aim of the present study was to evaluate the presence of IgA-NMDAR-Abs in the CSF of patients with IgG-NMDAR-Ab encephalitis and determine the potential association with a specific clinical pattern. The results suggested that although CSF IgA-NMDAR-Abs could be used as biological markers for the presence of ovarian teratoma, these markers do not have much influence on the clinical course of the disease.

METHODS Patients and NMDAR-Ab detection. We systematically tested 94 CSF samples of patients with NMDAR-Ab encephalitis ( 25 children [age $<12$ years] and 69 adult patients) diagnosed between October 2007 and February 2014 at the French Reference Centre on Paraneoplastic Neurological Syndrome for presence of IgA-NMDAR-Abs. This observational cohort study was based on patients diagnosed with NMDAR-Ab encephalitis using the CSF rather than the serum because of the better quality of the results and the observation of a 3\% false-positive rate of serum IgG-NMDAR-Abs in healthy patients. All analyzed CSF were the first available CSF obtained by a lumbar puncture realized for diagnostic purpose (median delay between the onset of the disease and the lumbar puncture: 30 days; interquartile range [IQR] 18-56) before any immunosuppressive treatment.

CSF IgG-NMDAR-Abs detection. For a diagnosis of NMDAR-Ab encephalitis, the CSF analysis had to fulfill the following previously established criteria for the presence of IgGNMDAR-Abs': (1) CSF samples must produce a specific pattern of neuropil immunostaining in the rat brain hippocampus; and (2) CSF samples must yield a positive cell-based assay (CBA) using HEK293 cells expressing both the GluN1 and GluN2B subunits of the NMDAR (figure 1).

For immunohistochemistry, rat brains were fixed in $4 \%$ paraformaldehyde for 1 hour at $4^{\circ} \mathrm{C}$, washed in $0.1 \mathrm{M}$ phosphate buffer, placed in sucrose $30 \%$ at $4^{\circ} \mathrm{C}$ until brain sink, and frozen. Twelve-micrometer-thick frozen rat brain sections were realized and were serially incubated with phosphate-buffered saline (PBS) for 10 minutes, in PBS containing 3\% normal goat serum and $3 \%$ bovine serum albumin for 1 hour and the patient's CSF (1:10) overnight at room temperature. The bound patient antibodies were visualized using Alexa488-conjugated anti-human IgG (Life Technologies, St. Aubin, France) diluted at 1/1,500 at room temperature for 1 hour and fluorescence microscopy (Axiophot; Zeiss, Oberkochen, Germany).

CBA was performed on HEK cells overexpressing the GluN1 (fused with the green fluorescent protein) and the GluN2b subunits of the NMDAR. Cells, fixed with $4 \%$ paraformaldehyde, were permeabilized in PBS containing $0.2 \%$ gelatin and $0.1 \%$ triton and then incubated with the patient's CSF (1:10) for 90 minutes. The bound antibodies were visualized using Cy3conjugated anti-human IgG (Cy ${ }^{\mathrm{TM}} 3$ AffiniPure Goat AntiHuman IgG; Jackson ImmunoResearch, West Grove, PA).

CSF IgA-NMDAR-Ab detection. Ninety-four CSF of patients with NMDAR-Ab encephalitis were available to study the presence of IgA-NMDAR-Ab. In order to detect such autoantibodies, we used the same CBA described above using a
Cy3-conjugated anti-human IgA (Jackson ImmunoResearch). For all positive patients by CBA, we controlled IgA-NMDAR-Abs immunostained rat brain hippocampus with a specific pattern of neuropil (figure 1). Absence of cross-reactivity between the secondary anti-human IgA and $\operatorname{IgG}$ was checked on purified patient IgG as previously described. ${ }^{2}$

Titration of CSF Ig-NMDAR-Abs. IgG-NMDAR-Ab and IgA-NMDAR-Ab titers were determined using a CBA and serial dilutions of CSF until the reactivity was no longer detectable. The assigned titer value is indicative of the last dilution in which the positivity was detected. Two investigators independently evaluated the endpoint dilution (C.B. and V.R.).

Detection of intrathecal antibody synthesis. The IgG, IgA, and albumin concentrations in the CSF and serum were evaluated using nephelometry (IMMAGE Immunochemistry Systems; Beckman-Coulter, Hialeah, FL). The CSF/serum albumin quotient (QAlb) was used to evaluate the integrity of the CSFblood barrier. The QAlb is age-dependent: the upper reference limit is calculated for each patient. ${ }^{10}$

To characterize intrathecal immunoglobulin synthesis, the immunoglobulins quotient $\left(\mathrm{QIg}=\left[\mathrm{Ig}_{\mathrm{CSF}} / \mathrm{Ig}_{\text {serum }}\right]\right)$ was calculated and compared to the corresponding QLim. QLim represents the maximum QIg that can be expected at a given QAlb in the absence of intrathecal immunoglobulin synthesis. ${ }^{10}$

Standard protocol approval, registration, and patient consent. Written consent was obtained from all patients, and this study was approved by the institutional review board of the University Claude Bernard Lyon 1 and Hospices Civils de Lyon. The biological samples were obtained from NeuroBioTec Hospices Civils de Lyon BRC (France, AC-208-73 NFS96-900).

Definition of clinical events, ancillary tests, treatment, and evolution. Detailed clinical data on acute disease stages were obtained at the time of biological diagnosis, and data regarding disease clinical course were collected during follow-up examinations. Clinical events classification was detailed previously. ${ }^{11}$

Prodromal symptoms were considered to be signs or symptoms arising prior to the first neurologic symptoms. These symptoms were subdivided into the following subgroups: (1) headache, (2) gastrointestinal symptoms, (3) infection signs, and (4) other. The first neurologic symptoms were considered to be the first clinical signs after the prodromal state, when present, or the first neurologic symptoms. The subsequent symptoms were clinical signs that arose after the first symptoms, with a delay interval of at least 24 hours. All symptoms were categorized into the following subgroups: (1) behavioral and psychiatric features, (2) seizure, (3) cognitive dysfunction (which included anterograde amnesia, speech disorder, and alteration of mental status), (4) movement disorders, (5) fluctuating level of consciousness, (6) dysautonomia, and (7) a subgroup called other for symptoms that did not correspond to any of the previously described features. The results from the initial ancillary examinations (MRI, CSF analysis, EEG, and tumor screening) were also compiled. Follow-up information was collected at regular intervals (3, 6, $9,12,18$, and 24 months) after diagnosis. Neurologic disability was assessed using the modified Rankin Scale (mRS). Recovery was defined as occurring when the patient's $\mathrm{mRS}$ score was determined to be 0,1 , or 2 (mRS $0-2$ ).

Teratoma samples analysis. When an associated teratoma was diagnosed, paraffin-embedded samples were collected. Fourmicrometer-thick sections were cut and stained with hematoxylinphloxine-saffron. A reference pathologist (I.T.) assessed the composition of each teratoma in different tissues, particularly the 


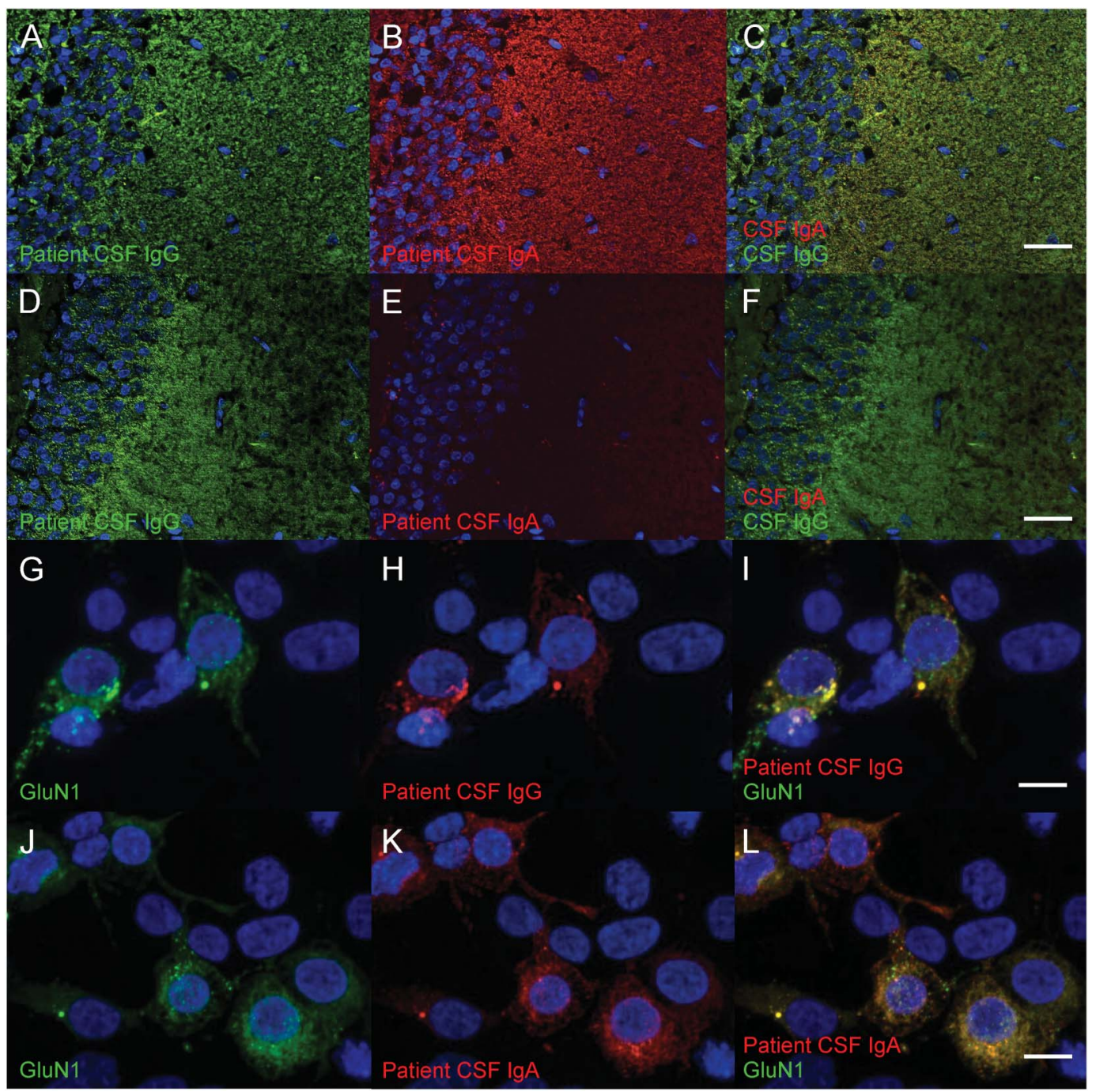

Rat hippocampal dentate gyrus neuropils were stained with patient CSF; the reactivity was revealed using anti-human immunoglobulin $\mathrm{G}(\mathrm{IgG})$ (in green) or immunoglobulin $\mathrm{A}$ (IgA) (in red) $(A-F$, scale bar $=50 \mu \mathrm{m})$. The CSF of the first patient (A-C) contained both IgG-NMDA receptor (NMDAR)-antibodies (Abs) (A) and IgA-NMDAR-Abs (B), both showing the same pattern of neuropil staining (C). The CSF of the second patient (D-F) only contained IgG-NMDAR-Abs (D). No IgA-NMDARAbs was detected (E, F). Human epithelial kidney (HEK) cells were transfected to express green fluorescent protein (GFP)tagged GluN1 (green; $G, J$ ) and were then incubated with CSF from patients; the reactivity was revealed using anti-human $\operatorname{lgG}($ red; H) or anti-human IgA (red; K) (G-L, scale bars $=10 \mu \mathrm{m})$. (I, L) The merged image of GFP-GluN1 and IgG or IgA reactivity, respectively. The nuclei were counterstained with 4',6-diamidino-2-phenylindole (DAPI).

nervous system parenchyma and respiratory and digestive mucosae (sites of possible IgA production).

Statistical analysis. Statistical analysis was performed using the $\mathrm{R}$ software version 3.1 .1 (http://www.r-project.org/). The $p$ values for demographic information, symptoms, ancillary tests results, and the clinical severity assessment (mRS, intensive care unit $[\mathrm{ICU}]$ admission, death) were obtained using the Fisher exact test for contingency tables with Bonferroni correction for multiple testing. A multivariate analysis was performed using the logistic regression technique including variables with a $p$ value $<0.05$ before Bonferroni adjustment. The logistic mixed model was fitted using the package lme4 version 1.1-7 (http://CRAN. R-project.org/package $=$ lme4). Comparisons of the medians and NMDAR-Ab titers were performed using the Wilcoxon rank-sum test (Mann-Whitney test). Because of a skewed distribution, we used a $\log$ transformation for the titers. A multivariate linear regression model, including the presence of a teratoma as cofactor, was used to assess the relationship between CSF IgANMDAR-Ab titers and CSF IgG-NMDAR-Ab titers. Analysis of factors affecting outcome (recovery defined as mRS 0-2) was performed using a logistic binary mixed model including a random effect per patients (on the intercept only) and 4 fixed effects (sex, CSF IgA-NMDAR-Ab status, ICU admission, and time [modeled as a quadratic function]).

RESULTS Detection of CSF IgA-NMDA-Abs and associated clinical presentation. The cohort of 94 IgG-NMDAR-Ab encephalitis patients (median age 19 years, range $1-76$ ) with available CSF comprised 25 children (median age 6 years, range 1-12; 17 girls 
Table Comparison of the clinical presentation of the 39 IgA-NMDAR-Abpositive patients with NMDAR-Ab encephalitis to the 55 IgA-NMDAR-Abnegative patients

\begin{tabular}{|c|c|c|c|}
\hline & $\begin{array}{l}\text { IgA-NMDAR-Ab- } \\
\text { positive } \\
(\mathrm{n}=39)\end{array}$ & $\begin{array}{l}\text { IgA-NMDAR-Ab- } \\
\text { negative } \\
\text { (n= 55) }\end{array}$ & $p$ Value \\
\hline Age, y, median (range) & $22(2-76)$ & $17(1-66)$ & NS \\
\hline Women & $34(87)$ & $44(80)$ & NS \\
\hline Children (age $<12$ y) & $6(15)$ & 19 (34) & NS \\
\hline Caucasian & $29(74)$ & $33(60)$ & NS \\
\hline \multicolumn{4}{|l|}{ Prodromal symptoms ${ }^{a}$} \\
\hline Assessable cases & 37 & 51 & \\
\hline Podroms & $20(54)$ & $26(51)$ & NS \\
\hline Headache & $13(35)$ & 9 (17) & NS \\
\hline Infectious diseases & $8(21)$ & $11(21)$ & NS \\
\hline Gastrointestinal signs & $6(16)$ & 4 (8) & NS \\
\hline Others ${ }^{b}$ & $5(13)$ & $3(6)$ & NS \\
\hline \multicolumn{4}{|l|}{ Oncologic status } \\
\hline Assessable cases & 38 & 55 & \\
\hline Tumors & $18(47)$ & $3(5)$ & $<0.001$ \\
\hline Ovarian teratoma & $17(44)$ & $3(5)$ & $<0.001$ \\
\hline Other ${ }^{c}$ & $1(2)$ & $0(0)$ & NS \\
\hline \multicolumn{4}{|l|}{ First symptoms ${ }^{a}$} \\
\hline Assessable cases & 39 & 54 & \\
\hline Behavior and psychiatric features & $24(61)$ & $23(43)$ & NS \\
\hline Seizures & $10(26)$ & $24(44)$ & NS \\
\hline Cognitive dysfunction & $12(30)$ & $10(18)$ & NS \\
\hline Anterograde amnesia & $3(7)$ & 1 (2) & NS \\
\hline Speech disorders & $3(7)$ & $4(7)$ & NS \\
\hline Alteration of mental status & $8(20)$ & $6(14)$ & NS \\
\hline Movement disorders & $0(0)$ & 2 (3) & NS \\
\hline Others $^{d}$ & $3(8)$ & $6(11)$ & NS \\
\hline \multicolumn{4}{|l|}{ Subsequent symptoms ${ }^{a}$} \\
\hline Assessable cases & 39 & 53 & \\
\hline Behavior and psychiatric features & $30(77)$ & $45(84)$ & NS \\
\hline Seizures & $26(67)$ & $46(86)$ & NS \\
\hline Cognitive dysfunction & $38(97)$ & $50(94)$ & NS \\
\hline Anterograde amnesia & $27(69)$ & $28(52)$ & NS \\
\hline Speech disorders & $21(53)$ & $34(64)$ & NS \\
\hline Alteration of mental status & $28(71)$ & $35(66)$ & NS \\
\hline Movement disorders & $30(77)$ & $34(64)$ & NS \\
\hline Fluctuating level of consciousness & $26(67)$ & $33(62)$ & NS \\
\hline Dysautonomia & $22(56)$ & $17(32)$ & NS \\
\hline Other symptoms ${ }^{e}$ & $7(18)$ & 10 (19) & NS \\
\hline \multicolumn{4}{|l|}{ Paraclinic examinations (acute phase) $^{\mathrm{a}}$} \\
\hline \multicolumn{4}{|l|}{ MRI } \\
\hline Assessable cases & 36 & 49 & \\
\hline Abnormal & 14 (39) & $17(35)$ & NS \\
\hline
\end{tabular}

and 8 boys), 61 female participants (median age 21 years, range 14-48), and 8 men (median age 33 years, range 22-76). The CSF samples obtained from these 94 individuals showed that 39 individuals (41\%) were positive for IgA-NMDAR-Abs (figure 1). Among the 39 IgA-NMDAR-Ab-positive patients, 30 patients were adult women, 3 patients were adult men, and 6 patients were children (age $<12$ years; 4 girls and 2 boys). We compared the 39 IgA-NMDAR-Ab-positive patients with the 55 IgA-NMDAR-Ab-negative patients identified in the cohort (table). The median age, sex ratio, and percentage with Caucasian origin were similar in both groups. The children tended to be underrepresented in the IgA-NMDAR-Ab-positive group (15\% vs $34 \%$ in the IgA-NMDAR-Ab-negative group, without reaching significance after Bonferroni adjustment). The incidences of prodromal signs and the first symptoms were similar in both groups. The complete clinical pattern was also similar in both groups. The incidence of abnormalities in ancillary tests (MRI, EEG, and CSF) and the clinical severity ( $\mathrm{mRS}$ at onset, ICU admission, death) were similar in both populations. Ninety-one patients (39 IgANMDAR-Ab-positive and 52 IgA-NMDAR-Abnegative individuals) had a follow-up (median 24 months, range 3-24). The global progression and recovery were similar in the groups (figure 2). In multivariable analysis, no effect of the CSF IgANMDAR-Ab status on the time to recovery was identified.

Associated tumors. The positivity of CSF IgANMDAR-Abs is independently associated with the presence of an ovarian teratoma (odds ratio 10.59, confidence interval 2.81-52.88; $p=0.0012$ ). Approximately half of the IgA-NMDAR-Ab-positive patients with a known oncologic status (18/38, 47\%) had a definite associated tumor, primarily ovarian teratomas $(17 / 18,94 \%)$, compared with 5\% (3/55) in the IgA-NMDAR-Ab-negative group $(p<0.001)$. In the adult women, ovarian teratomas were present in $17 / 29(58 \%)$ of the IgA-NMDAR-Ab-positive women vs $3 / 31(9.6 \%)$ of the IgA-NMDAR-Abnegative women $(p=0.001)$. The histologic nature of the ovarian cystic lesion observed through CT scan was unknown for one IgA-NMDAR-Ab-positive woman who died before the end of the diagnostic assessment for an ovarian tumor and surgical excision. Among the men, only one individual had a tumor (a perineal schwannoma) ${ }^{11}$ and was positive for IgA-NMDAR-Abs. Among the children, no underlying tumor was detected in the groups (19 IgA-NMDAR-Ab-negative and 6 IgA-NMDAR-Abpositive children). In the adult female population at risk for ovarian teratoma, the CSF positivity of IgANMDAR-Ab detection showed $85 \%$ sensitivity, 


\begin{tabular}{|c|c|c|c|}
\hline Continued & & & \\
\hline & $\begin{array}{l}\text { IgA-NMDAR-Ab- } \\
\text { positive } \\
(\mathrm{n}=39)\end{array}$ & $\begin{array}{l}\text { IgA-NMDAR-Ab- } \\
\text { negative } \\
(\mathrm{n}=55)\end{array}$ & $p$ Value \\
\hline \multicolumn{4}{|l|}{ CSF } \\
\hline Assessable cases & 37 & 48 & \\
\hline Abnormal & $32(86)$ & $43(88)$ & NS \\
\hline \multicolumn{4}{|l|}{ EEG } \\
\hline Assessable cases & 33 & 48 & \\
\hline Abnormal & $31(94)$ & $42(87)$ & NS \\
\hline Epileptiform abnormalities ${ }^{f}$ & 7 (23) & 16 (38) & NS \\
\hline \multicolumn{4}{|l|}{ Clinical severity ${ }^{a}$} \\
\hline Assessable cases & 39 & 53 & \\
\hline ICU & 27 (69) & $32(60)$ & NS \\
\hline Death & 1 (3) & $1(2)$ & NS \\
\hline \multicolumn{4}{|l|}{ Initial mRS } \\
\hline mRS 0 & $0(0)$ & $0(0)$ & NS \\
\hline mRS 1-2 & $0(0)$ & $0(0)$ & NS \\
\hline mRS 3-4 & $9(23)$ & $18(35)$ & NS \\
\hline mRS 5 & $30(77)$ & $34(65)$ & NS \\
\hline
\end{tabular}

Abbreviations: $\mathrm{Ab}=$ antibody; ICU = intensive care unit; $\operatorname{lgA}=$ immunoglobulin $\mathrm{A} ; \mathrm{mRS}=$ modified Rankin Scale; NMDAR = NMDA receptor; NS = not significant.

Values are $n(\%)$ unless noted otherwise.

${ }^{a}$ No. on assessable cases.

${ }^{\mathrm{b}}$ Asthenia, anorexia, myalgia, cervicalgia, photophobia.

${ }^{\mathrm{c}}$ Perineal schwanomma.

${ }^{d}$ Diplopia, walking problem, upper limb and face pain, sleep disorder, gait ataxia.

${ }^{e}$ Walking problem, cerebellar syndrome, pyramidal syndrome, rigidity, neuroleptic malignant syndrome, nystagmus, acouphobia, sensory and motor disorder, myelitis.

${ }^{f}$ Clustered seizures, status epilepticus, critical activities.

$70 \%$ specificity, a $57 \%$ positive predictive value, and a $90 \%$ negative predictive value for the diagnosis of ovarian teratoma.

All 20 teratomas were detected after the diagnosis of encephalitis, except for the teratoma in one patient who was diagnosed after pelvic ultrasound examination with ovarian cysts of unknown nature at 3 years prior to the onset of encephalitis. The median time between the first neurologic symptom and the surgical removal of the teratoma was 32 days (range 6-386). This median delay was similar between the IgA-NMDARAb-positive and IgA-NMDAR-Ab-negative patients (34 days, range 6-386; and 30 days, range 10-83, respectively). In 2 patients, an early body CT scan did not detect a cystic ovarian tumor, which was subsequently diagnosed (a few days later) through pelvic MRI and endovaginal ultrasound, indicating estimated tumor sizes of 7 and $18 \mathrm{~mm}$. In 4 patients, teratomas were detected at several months (from 6 to 10 months) after the onset of neurologic symptoms and the diagnosis of the NMDAR-Ab encephalitis. However, 3 of them had a diagnostic assessment for an ovarian tumor during the first weeks of the disease; the assessment comprised at least one CT scan that was considered normal. The last patient did not have a paraclinical checkup before 10 months after the onset of the encephalitis. These 4 patients were IgA-NMDAR-Ab positive.

Histologic analysis of teratoma samples. Nineteen of the 20 diagnosed ovarian teratomas in the cohort were collected at the French Reference Centre on Paraneoplastic Neurological Syndrome. Sixteen tumors were from IgA-NMDAR-Ab-positive patients and 3 from IgA-NMDAR-Ab-negative patients. The mean tumor size was similar in the IgA-NMDAR-Ab-positive and IgA-NMDAR-Ab-negative patients $(2.71 \pm 1.20 \mathrm{~cm}$ and $2.16 \pm 1.26 \mathrm{~cm}$, respectively). Histologic examination of all cases showed teratomatous lesions composed of derivatives of all 3 germ cell layers. Two cases showed neuroepithelial foci corresponding to an immature glial component. A mature glial component was present in all cases. Among the 16 teratomas associated with IgA-NMDAR-Abs, 2 tumors were immature, and 13/16 tumors (81\%) contained mucosal epithelium (gastrointestinal [9/16] or respiratory [9/16] epithelium). The 3 teratomas not associated with IgA-NMDA-Abs were mature and contained mucosal epithelium (gastrointestinal [2/3] or respiratory [1/3]) (figure e-1 at Neurology.org/nn).

CSF NMDAR-Ab titers and intrathecal immunoglobulin synthesis. We examined the CSF IgG-NMDAR-Ab titers at diagnosis in 12 patients with IgANMDAR-Abs (6/12 with a teratoma) and 12 patients without IgA-NMDAR-Abs (2/12 with a teratoma). The CSF IgG-NMDAR-Ab titers were significantly higher in the patients positive for IgANMDAR-Abs (median 320; IQR [50-560] vs 40 [20-140], $p=0.0136$, Mann-Whitney test) and in the patients with a teratoma (320; IQR [100-640] vs $40[20-160], p=0.0081)$. The CSF IgA-NMDAR$\mathrm{Ab}$ titers were significantly lower than the CSF IgGNMDAR-Ab titers (median 30; IQR [12.5-80] vs 320 [50-560], $p=0.0037$ ). In IgA-NMDAR-Abpositive patients, the CSF IgA-NMDAR-Ab titers were linearly related to the CSF IgG-NMDAR-Ab titers $\left(r^{2}=0.54, p=0.012\right)$.

The IgG and IgA quotients were assessed in 4 patients with IgA-NMDAR-Abs. Two patients had an associated teratoma, while the other 2 patients did not. The QAlb was consistently inferior to the upper age-related reference, reflecting the integrity of the CSF-blood-brain barrier in all cases. Intrathecal $\operatorname{IgG}$ synthesis $(\mathrm{QIgG}>$ QLim) was detected in 3/4 patients. The only patient without IgG intrathecal synthesis had no associated teratoma. Intrathecal IgA synthesis (QIgA $>$ QLim) was only detected in the 2 patients without teratomas.

DISCUSSION The present study evaluates the presence of IgA-NMDAR-Abs in the CSF of patients 


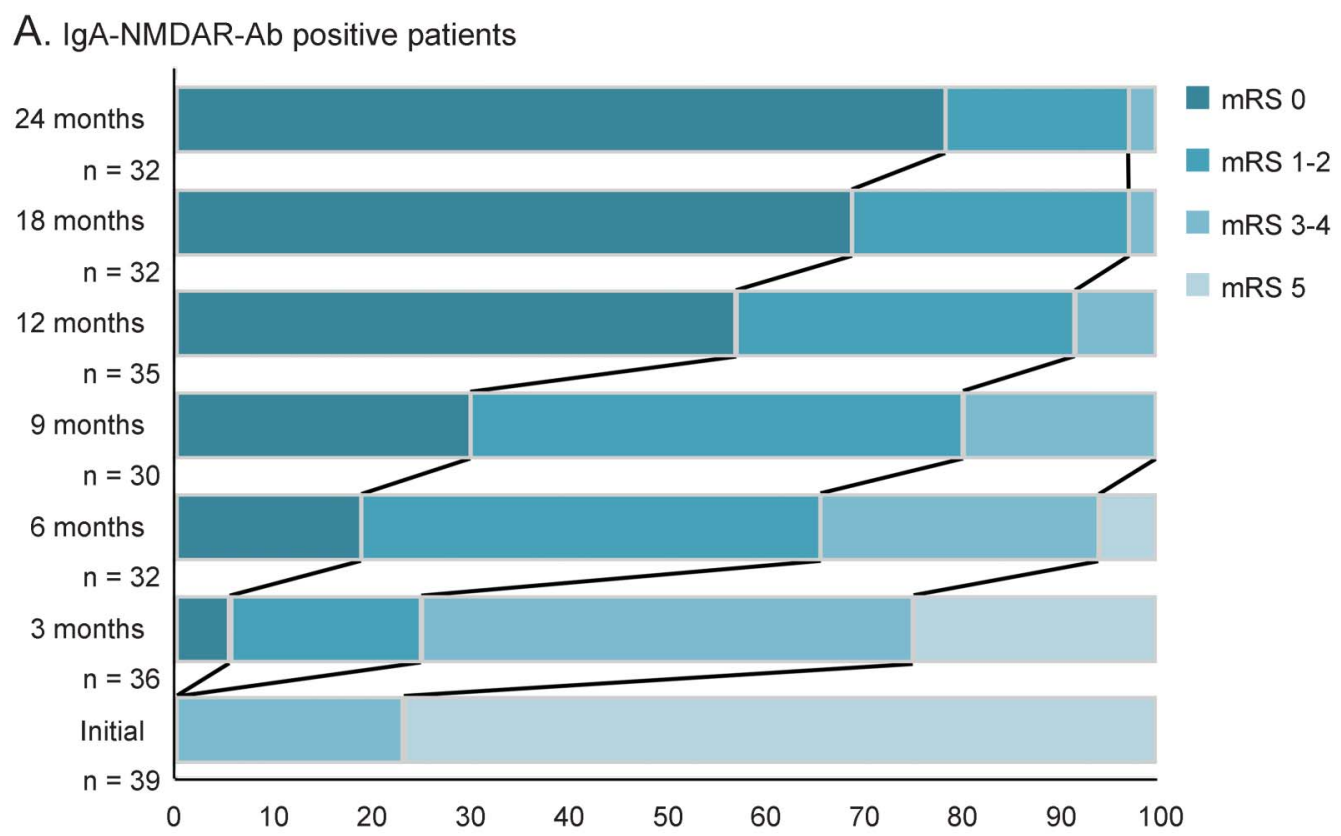

B. IgA-NMDAR-Ab negative patients

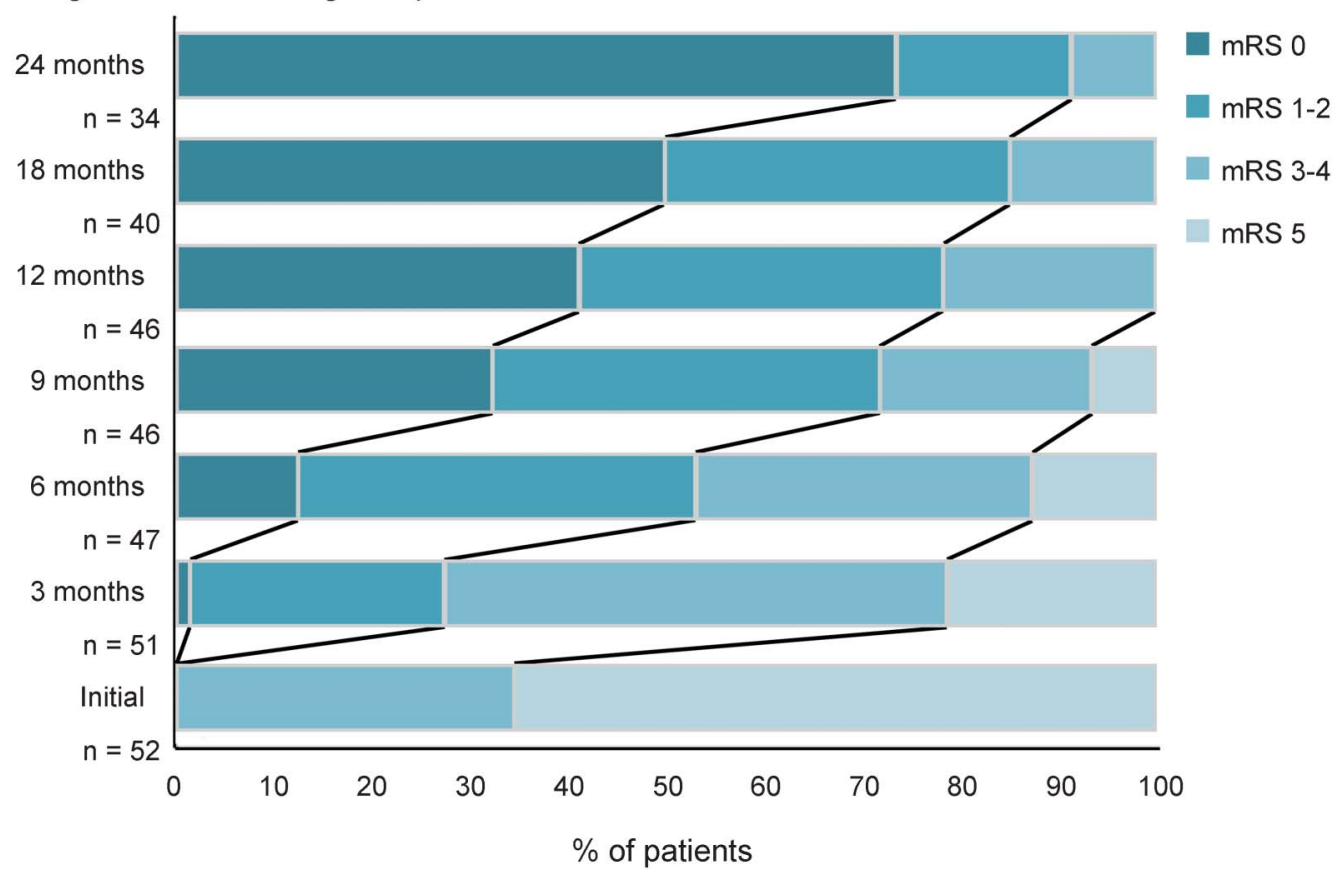

The clinical outcome was measured using the modified Rankin Scale (mRS) at 0, 3, 6, 9, 12, 18, and 24 months after the onset of NMDA receptor (NMDAR)-antibody (Ab) encephalitis in immunoglobulin A (IgA)-NMDAR-Ab-positive (A) and IgANMDAR-Ab-negative (B) patients.

with NMDAR-Ab encephalitis and determines their relative contribution to this syndrome. In the retrospective cohort, $41 \%$ of the patients with NMDAR-Ab encephalitis had both CSF IgG- and IgA-NMDAR-Abs. This CSF IgA-NMDAR-Abpositive rate was consistent with the $30 \%$ serologic IgA-NMDAR-Ab-positive rate previously reported in NMDAR-Ab encephalitis ${ }^{6}$ and markedly higher than the false-positive rate observed in large seroprevalence studies. ${ }^{7}$ No significant differences in clinical and paraclinical presentation, severity, and prognosis according to the IgA status were identified. No specific clinical features were associated with CSF IgA-NMDAR-Ab positivity, except that ovarian teratomas were much more frequent in patients with CSF IgA-NMDAR-Abs. These results suggest that CSF IgA-NMDAR-Abs could be biomarkers for the presence of an ovarian 
teratoma, without influencing the clinical course of this disease.

The absence of a clinical influence of IgANMDAR-Ab status is not in favor of a particular pathogenic role for IgA-NMDAR-Abs in the neurologic symptoms, although it was suggested by other authors. ${ }^{6}$ The specific role of $\operatorname{IgA}$ and $\operatorname{IgG}$ autoantibodies has previously been discussed in other autoantibody-mediated diseases. In the mucous membrane pemphigoid, the dual circulating antibody response with $\operatorname{IgA}$ in addition to $\operatorname{IgG}$ has been associated with a more severe disease, ${ }^{12}$ although studies have not confirmed the importance of IgA antibodies as a prognostic indicator. ${ }^{13}$ Thus, the clinical relevance of these IgA-Abs remains controversial, and if present, might be weak, with an uncertain pathogenesis.

The site of IgA production is another nonelucidated issue. The absence of intrathecal secretion of IgA-NMDAR-Abs in IgA-positive patients with a teratoma should be confirmed but could suggest a peripheral antibody response when a teratoma is present. One hypothesis could be that teratomas directly trigger the production of the IgA serotype of immunoglobulins in the periphery. We also observed that CSF IgA-NMDAR-Ab positivity was associated with higher CSF IgG-NMDAR-Ab titers. When available, CSF IgA-NMDAR-Ab titers seem to be proportional to the CSF IgG NMDAR-Ab titers. This result suggests that IgA status might primarily reflect the intensity of humoral autoimmunity against the NMDAR, and this response could be particularly activated in the periphery through the presence of teratomas. Indeed, previous studies have shown that patients with an ovarian teratoma have significantly higher titers of IgG-NMDAR-Abs than those of patients without teratoma. ${ }^{4}$ Nevertheless, we observed 3 patients with NMDAR-Ab encephalitis and a teratoma but without CSF IgA-NMDAR-Abs, suggesting that the humoral reaction might differ according to the patient. We did not observe any difference in the histopathologic examination of the teratoma according to the patient IgA status among the 19 analyzed teratomas. Specifically, the presence of gastrointestinal or respiratory mucosa in the teratoma was not sufficient to explain the observed IgA-NMDAR-Ab production. Thus, the teratoma-associated mechanism leading to IgA production remains unclear, and another external agent, such as a viral infection, could be involved.

A practical issue of the present study is the value of the CSF IgA-NMDAR-Ab status as an indicator of an associated teratoma in the context of NMDAR-Ab encephalitis. At the individual level, there is no available predictive surrogate marker for the association of an ovarian tumor or a primary tumor elsewhere. In the present study, initial radiologic examinations did not reveal an ovarian teratoma in 5 cases. In clinical practice, the detection of a small teratoma (mean tumor size of $2.3 \mathrm{~cm}$ in the present study) in a patient with a severe neurologic state requiring respiratory assistance and sedation is typically difficult but must be considered a high priority. Because of the observed $85 \%$ sensitivity and $90 \%$ negative predictive value in the adult female population at risk for ovarian teratomas, we suggest that CSF-IgA-NMDAR-Ab status could be a predictive indicator for managing the patient screening for an associated teratoma. For an IgA-NMDAR-Ab-positive woman, when early radiologic pelvic investigations are negative, a second screening with pelvic CT or MRI or endovaginal ultrasound should be conducted promptly and repeated if negative. Future prospective studies are needed to further demonstrate the usefulness of CSF IgA-NMDAR-Ab status as a biomarker for a teratoma in anti-NMDAR-Abs encephalitis.

\section{AUTHOR CONTRIBUTIONS}

Virginie Desestret: analysis or interpretation of the data, drafting or revising the manuscript for intellectual content. Aude Chedeville: acquisition and analysis of data. Aurélien Viaccoz: analysis and interpretation of the data. Chloé Bost: acquisition and analysis of data. François Ducray: drafting or revising the manuscript for intellectual content. Géraldine Picard: acquisition and analysis of the data. Véronique Rogemond: acquisition, analysis, or interpretation of the data. Marie-Océane Chaffois: acquisition and analysis of the data. Charlotte Blanc: acquisition and analysis of the data. Claire Bardel: statistical analysis of data. Isabelle Treilleux: analysis and interpretation of data. Olivier Pascual: drafting or revising the manuscript for intellectual content. Jean-Christophe Antoine: drafting or revising the manuscript for intellectual content. Jean-Yves Delattre: drafting or revising the manuscript for intellectual content. Jérôme Honnorat: analysis or interpretation of the data, design or conceptualization of the study, drafting or revising the manuscript for intellectual content.

\section{ACKNOWLEDGMENT}

The authors thank Julien Bancel (Immunology Laboratory, Hôpital E. Herriot, Lyon, France) for providing guidance and advice.

\section{STUDY FUNDING}

Supported by a grant from the Fédération pour la Recherche sur le Cerveau (FRC).

\section{DISCLOSURE}

V. Desestret, A. Chefdeville, A. Viaccoz, C. Bost, F. Ducray, G. Picard, V. Rogemond, M.-O. Chaffois, and C. Blanc report no disclosures. C. Bardel received research support from La Ligue Contre le Cancer. I. Treilleux and O. Pascual report no disclosures. J.-C. Antoine received travel funding from le Laboratoire Francais des Biotechnologies et du Fractionnement, le CSL Behring, Genzyme, and is an associate editor for Revue Neurologique. J.-Y. Delattre is on the editorial board for The Oncologist and received research support from Institut National du Cancer, Ligue Nationale Contre le Cancer. J. Honnorat reports no disclosures. Go to Neurology.org/nn for full disclosures.

Received July 14, 2015. Accepted in final form September 9, 2015.

\section{REFERENCES}

1. Hughes EG, Peng X, Gleichman AJ, et al. Cellular and synaptic mechanisms of anti-NMDA receptor encephalitis. J Neurosci 2010;30:5866-5875. 
2. Mikasova L, De Rossi P, Bouchet D. Disrupted surface cross-talk between NMDA and Ephrin-B2 receptors in anti-NMDA encephalitis. Brain 2012;135: 1606-1621.

3. Planaguma J, Leypoldt F, Mannara F, et al. Human $\mathrm{N}$-methyl D-aspartate receptor antibodies alter memory and behaviour in mice. Brain 2014;138:94-109.

4. Gresa-Arribas N, Titulaer MJ, Torrents A, et al. Antibody titres at diagnosis and during follow-up of anti-NMDA receptor encephalitis: a retrospective study. Lancet Neurol 2014;13:167-177.

5. Lancaster E, Leypoldt F, Titulaer MJ, et al. IgG antibodies to the NMDA receptor are distinct from IgA and IgM responses. Ann Neurol 2015;77:183.

6. Prüss $\mathrm{H}, \mathrm{Klingbeil} \mathrm{C,} \mathrm{Probst} \mathrm{C,} \mathrm{et} \mathrm{al.} \mathrm{IgA} \mathrm{NMDA} \mathrm{receptor}$ antibodies are markers of synaptic immunity in slow cognitive impairment. Neurology 2012;78:1743-1753.

7. Dahm L, Ott C, Steiner J, et al. Seroprevalence of autoantibodies against brain antigens in health and disease. Ann Neurol 2014;76:82-94.
8. Doss S, Wandinger KP, Hyman BT, et al. High prevalence of NMDA receptor IgA/IgM antibodies in different dementia types. Ann Clin Transl Neurol 2014;1:822-832.

9. Dalmau J, Gleichman AJ, Hughes EG, et al. Anti-NMDAreceptor encephalitis: case series and analysis of the effects of antibodies. Lancet Neurol 2008;7:1091-1098.

10. Reiber H, Peter JB. Cerebrospinal fluid analysis: diseaserelated data patterns and evaluation programs. J Neurol Sci 2001;184:101-122.

11. Viaccoz A, Desestret V, Ducray F, et al. Clinical specificities of adult male patients with NMDA receptor antibodies encephalitis. Neurology 2014;82:556-563.

12. Setterfield J, Shirlaw PJ, Kerr-Muir M, et al. Mucous membrane pemphigoid: a dual circulating antibody response with $\operatorname{IgG}$ and $\operatorname{IgA}$ signifies a more severe and persistent disease. Br J Dermatol 1998;138:602-610.

13. Cozzani E, Drosera M, Parodi A, Carrozzo M, Gandolfo S, Rebora A. Frequency of IgA antibodies in pemphigus, bullous pemphigoid and mucous membrane pemphigoid. Acta Derm Venereol 2004;84:381-384. 


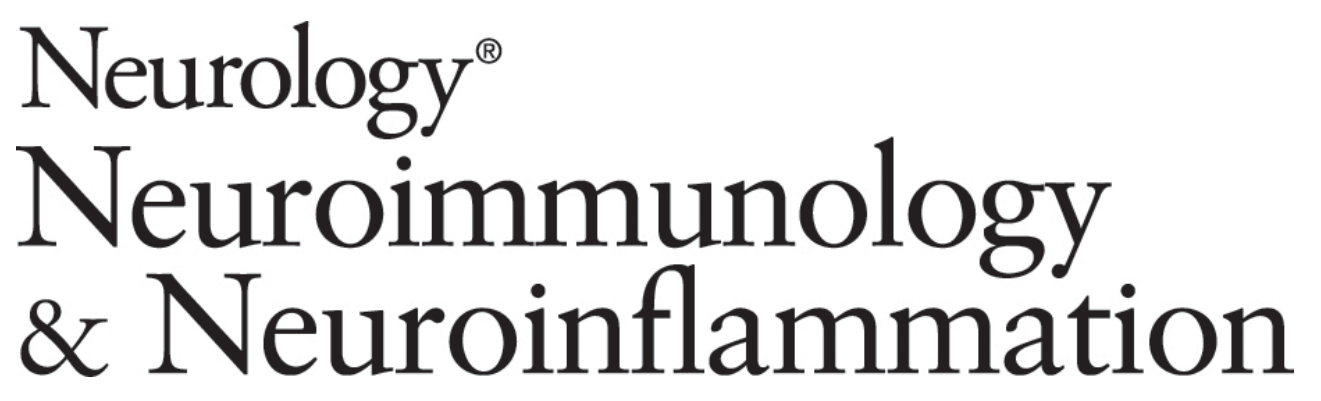

CSF IgA NMDAR antibodies are potential biomarkers for teratomas in anti-NMDAR encephalitis

Virginie Desestret, Aude Chefdeville, Aurélien Viaccoz, et al.

Neurol Neuroimmunol Neuroinflamm 2015;2;

DOI 10.1212/NXI.0000000000000166

This information is current as of October 29, 2015

Neurol Neuroimmunol Neuroinflamm is an official journal of the American Academy of Neurology.

Published since April 2014, it is an open-access, online-only, continuous publication journal. Copyright $\odot$ 2015 American Academy of Neurology. All rights reserved. Online ISSN: 2332-7812.

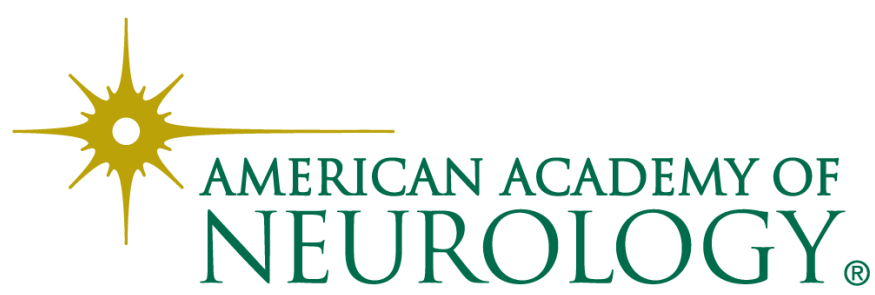




\section{Updated Information \& Services}

\section{Supplementary Material}

\section{References}

Citations

Subspecialty Collections

Permissions \& Licensing

Reprints including high resolution figures, can be found at: http://nn.neurology.org/content/2/6/e166.full.html

Supplementary material can be found at: http://nn.neurology.org/content/suppl/2015/10/29/2.6.e166.DC1

This article cites 13 articles, 1 of which you can access for free at: http://nn.neurology.org/content/2/6/e166.full.html\#\#ref-list-1

This article has been cited by 1 HighWire-hosted articles: http://nn.neurology.org/content/2/6/e166.full.html\#\#otherarticles

This article, along with others on similar topics, appears in the following collection(s):

\section{Autoimmune diseases}

http://nn.neurology.org//cgi/collection/autoimmune_diseases

\section{Encephalitis}

http://nn.neurology.org//cgi/collection/encephalitis

Paraneoplastic syndrome

http://nn.neurology.org//cgi/collection/paraneoplastic_syndrome

Information about reproducing this article in parts (figures,tables) or in its entirety can be found online at:

http://nn.neurology.org/misc/about.xhtml\#permissions

Information about ordering reprints can be found online:

http://nn.neurology.org/misc/addir.xhtml\#reprintsus

Neurol Neuroimmunol Neuroinflamm is an official journal of the American Academy of Neurology.

Published since April 2014, it is an open-access, online-only, continuous publication journal. Copyright $\odot$ 2015 American Academy of Neurology. All rights reserved. Online ISSN: 2332-7812.

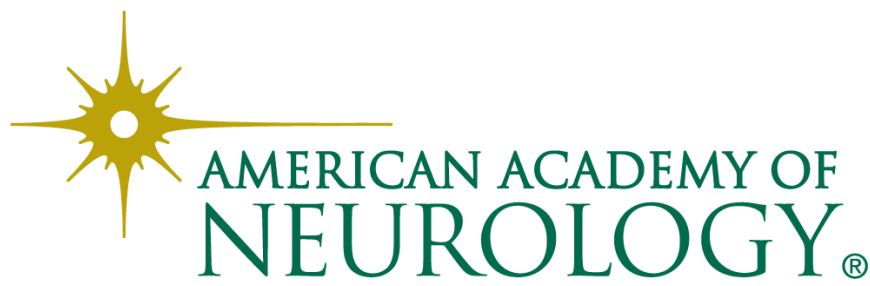

